\title{
Lipoprotein and Apoprotein Profiles of Hyperlipidemic Atherosclerosis-Prone Japanese Quail
}

\author{
Junichi Nagata,* Goki Maeda, Hirosuke OKu, \\ Takayoshi ToDA, ${ }^{1}$ and Isao CHINEN \\ Laboratory of Applied Biochemistry, Faculty of Agriculture, and ${ }^{1}$ Clinical \\ Laboratory Department, University of the Ryukyus, Okinawa 903-01, Japan
}

(Received June 2, 1996)

Summary The purpose of this study was to characterize the lipoprotein and apoprotein profiles of hyperlipidemic atherosclerosis-prone (HAP) Japanese quail. HAP and commercially available (CA) Japanese quail were fed either a semi-purified diet containing $1 \%$ cholesterol or a cholesterol-free diet for two weeks. The lipoproteins of CA and HAP quail fed cholesterol-free diet were composed of two fractions: densities ranging from 1.02 to 1.09 and from 1.09 to 1.21 . The lipoprotein distribution patterns obtained from both strains showed an HDL-predominant pattern. A protein of $26 \mathrm{kDa}$ was the major apoprotein in the entire density range of the lipoprotein class. Marked increases in the cholesterol ester levels were observed in the lower density fractions $(1.006<d<$ 1.055: chylomicron and VLDL fractions) of the cholesterol-fed quail, accounting for $46 \%$ of the total lipids in CA quail and 54\% in HAP quail. In addition, the presence of a protein of $470 \mathrm{kDa}$ was exclusively observed in the lower density fractions $(1.006<d<1.055)$ of the cholesterol-fed HAP quail. The fatty-acid compositions of the chylomicron and VLDL fractions were affected by the dietary cholesterol in both strains: a decrease in concentration of 16:0 and increase in 18:0 (weight \%). However, cholesterol feeding had no effect on the level of cholesterol, chemical composition or fatty-acid composition of the HDL fractions in either strain. Although the lipoprotein and apoprotein profiles of HAP quail showed resemblances to those of the CA quail, expression of the 470 $\mathrm{kDa}$ protein in the lipoproteins $(d<1.055)$ appeared to be pronounced in HAP quail. The relevance of these lipoprotein and apoprotein profiles to the genesis of atherosclerosis was discussed in this study.

Key Words quail, lipoprotein, cholesterol metabolism

Serum cholesterol level is positively correlated with the risk of coronary heart disease (CHD) (1), and an increased level results in the genesis of atherosclerosis

* To whom correspondence should be addressed. 
in many species (2). It is well-known that the hyperlipidemia influence lipoprotein distributions and apoprotein profiles. An elevated level of low-density lipoproteins (LDL) and reduced level of high-density lipoproteins (HDL) are associated with atherosclerosis in humans and experimental animals (3). Thus, lipoproteins play a vehicle role for the transport of serum cholesterol, phospholipid and triglyceride (4, 5). Apoproteins are also responsible for the receptor-mediated metabolism of cholesterol (6). Therefore, it is important to elucidate their associations with the disease because the metabolic fate of lipoproteins or apoproteins may bear relevance for the etiology of CHD.

Quail have been used as model for the investigation of atherosclerosis in our laboratory (7). Recently, our co-workers established an atherosclerosis-susceptible strain which develops hypercholesterolemia and atherosclerosis in response to dietary cholesterol. Although $\mathrm{Wu}$ and Donaldson reported that the diet-dependent increase of serum cholesterol level was largely associated with the low-density lipoprotein fractions $(d<1.063)$ of quail $(8)$, few studies have focused on the lipoprotein and apoprotein profiles of susceptible quail.

In this study, we have investigated, in detail, the effect of cholesterol on the lipoprotein distributions and apoprotein profiles of hyperlipidemic atherosclerosisprone (HAP) quail in an attempt to elucidate the cholesterol metabolism of HAP quail.

\section{MATERIALS AND METHODS}

Animals and diets. Male commercially available (CA) Japanese quail, 17weeks-old, were purchased from Japan SLC, Inc. (Shizuoka, Japan). Male HAP Japanese quail were developed and supplied by Otska Pharmaceutical Co., Ltd. (Tokyo, Japan). Ryukyu University's guide for the care and use of laboratory animals was followed in this study. Animals were housed individually under controlled room temperature $\left(25 \pm 2{ }^{\circ} \mathrm{C}\right)$ and a light/dark cycle (light was on from 0800 to 2000 hours). Both strains were divided into two groups and fed a semi-purified diet containing either $1 \%$ cholesterol or no cholesterol. The diet composition in grams per $100 \mathrm{~g}$ diet was as follows: 20 casein (Wako Pure Chemicals Co. Ltd., Osaka, Japan); 10 corn oil; 4 mineral mixture (Oriental Yeast, Tokyo, Japan); 1 vitamin mixture (Oriental Yeast); 5 cellulose powder; 1 cholesterol or 0 cholesterol; and $\sim 100 \beta$-corn starch. Quail were fed the semi-purified diet ad libitum for two weeks. At the end of the experimental period, the quail were fasted overnight and sacrificed by decapitation.

Lipoprotein separation. Serum was obtained by centrifugation of blood at $3,000 \mathrm{rpm}$ for $20 \mathrm{~min}$. Serum specimens separated from individual animals were combined for each group and used to isolate the lipoproteins. The procedure was described previously (9). Briefly, to investigate the distribution of the HAP quail lipoproteins, two types of density gradient were constructed with $\mathrm{NaCl}$ and $\mathrm{KBr}$; one gradient range was from $d=1.04$ to $d=1.25$, and the other was less than $d=$ 
1.04. Ultracentrifugation was carried out by use of a RP-80AT rotor in a Hitachi $\mathrm{CP}-100$ at $80,000 \mathrm{rpm}(322,000 \times g)$ for $8 \mathrm{~h}$ at $15^{\circ} \mathrm{C}$. After completion of the centrifugation, fractions of $0.1 \mathrm{ml}$ were successively collected from the tube bottom. Lipoprotein distributions were determined on the basis of cholesterol concentration in the fractionated sample.

Lipid analyses. Lipoprotein lipids were determined enzymatically using a commercial kit (Wako Pure Chemicals). The liver lipids were extracted by chloroform-methanol $(2: 1, \mathrm{v} / \mathrm{v})$. Cholesterol, triglyceride and phospholipid were assayed as described elsewhere (10).

SDS-polyacrylamide gel electrophoresis. Molecular weight and apolipoprotein composition were measured by SDS-polyacrylamide gel electrophoresis (SDSPAGE). The isolated lipoproteins were solubilized directly in 20\% SDS and applied on SDS-PAGE gel (3 or 12\% polyacrylamide gel containing 1\% SDS and 4\% mercaptoethanol. Gels were stained with Coomassie brilliant blue R-250, and scanned by a EPA-3000 densito-pattern analyzer (Maruzen Petrochemical Co., Ltd., Tokyo, Japan) at $650 \mathrm{~nm}$ wavelength. Apoprotein compositions were estimated by the densitometric intensity of the stained band.

Fatty-acid composition of lipoprotein. The total lipids extracted from the lipoproteins with chloroform-methanol $(2: 1, \mathrm{v} / \mathrm{v})$ were directly trans-methylated by the use of a boron trifluoride methanol complex. Methylated fatty acid was analyzed by gas-liquid chromatography (Shimadzu 14A gas chromatograph: Shimadzu Co., Ltd., Kyoto, Japan) on a fused silica capillary column (CBP20-M25025, Shimadzu Co., Ltd.). The oven temperature was programmed from a hold at $50^{\circ} \mathrm{C}$ for $1 \mathrm{~min}$, raised to $100^{\circ} \mathrm{C}$ at a rate of $10^{\circ} \mathrm{C} / \mathrm{min}$, and thereafter to $200^{\circ} \mathrm{C}$ at a rate of $3^{\circ} \mathrm{C} / \mathrm{min}$. The temperature for the detector and injection port was $230^{\circ} \mathrm{C}$. The identification of fatty acid methyl esters was done by gas chromatography mass spectrometry (Shimadzu QP 1000EX).

Statistical analysis. Data were presented as $\mathbf{M} \pm \mathbf{S E M}$ (standard error of the mean) unless otherwise stated. The statistical significances of the effects of cholesterol (fed vs. unfed) and strain (CA vs. HAP) were assessed by two way analysis of variance (11), followed by the inspection of differences between pairs of means. Statistical significance was reached at $p<0.01$ or 0.05 .

\section{RESULTS}

All animal groups showed similar growth rates, suggesting a comparable dietary intake (Table 1). The increase of liver weight induced by cholesterol feeding was statistically significant $(p<0.05)$ in both strains. These observations roughtly agree with the results obtained in a previous investigation.

Table 2 lists the concentrations of serum and liver lipids. The recovery of lipoprotein lipids on ultracentrifugation showed about $55 \%$ of variations. In this study, therefore, serum lipid levels were expressed as the sum of the seprated lipoprotein lipids. The feeding of cholesterol induced hyperlipidemia in both strins. 
Table 1. Growth parameter.

\begin{tabular}{lcccc}
\hline & CA $(-)$ & CA $(+)$ & HAP $(-)$ & HAP $(+)$ \\
\hline Food intake $(\mathrm{g} /$ day $)$ & $9.2 \pm 0.9$ & $9.1 \pm 0.4$ & $7.5 \pm 0.8$ & $8.3 \pm 0.4$ \\
Final body weight $(\mathrm{g})$ & $101 \pm 1.9$ & $102 \pm 1.9$ & $108 \pm 2.5$ & $106 \pm 3.6$ \\
Liver weight $(\mathrm{g} / 100 \mathrm{~g}$ body weight $)$ & $1.74 \pm 0.1^{\mathrm{a}}$ & $1.97 \pm 0.2^{\mathrm{a}}$ & $1.28 \pm 0.0^{\mathrm{b}}$ & $1.71 \pm 0.2^{\mathrm{b}}$ \\
\hline
\end{tabular}

Data show M \pm SEM of 10 quail. Same superscript letters show statistically significant difference at $p<0.05$. CA, commercially available Japanese quail; HAP, hyperlipidemic atherosclerosis-prone Japanese quail; - , cholesterol-free;,$+ 1 \%$ cholesterol.

Table 2. Lipid concentrations of serum and liver.

\begin{tabular}{lcccc}
\hline & CA $(-)$ & CA $(+)$ & HAP $(-)$ & HAP $(+)$ \\
\hline Serum (mg/dl) & & & & \\
Total cholesterol & 63.8 & 286 & 117 & 1,470 \\
Cholesterol ester & 51.6 & 209 & 84.8 & 1,117 \\
Triglyceride & 32.2 & 66.6 & 43.4 & 220 \\
Phospholipid & 89.0 & 136 & 135 & 426 \\
Liver (mg/g) & & & & \\
Total cholesterol & $4.86 \pm 0.44^{\mathrm{a}}$ & $26.2 \pm 3.2^{\mathrm{aA}}$ & $8.66 \pm 1.91^{\mathrm{b}}$ & $48.2 \pm 3.3^{\mathrm{bA}}$ \\
Cholesterol ester & $1.33 \pm 0.13^{\mathrm{a}}$ & $17.6 \pm 1.6^{\mathrm{aA}}$ & $5.18 \pm 1.67^{\mathrm{b}}$ & $38.6 \pm 2.7^{\mathrm{bA}}$ \\
Triglyceride & $16.1 \pm 2.9^{\mathrm{A}}$ & $16.7 \pm 2.8$ & $10.8 \pm 1.5^{\mathrm{A}}$ & $12.9 \pm 1.6$ \\
Phospholipid & $31.3 \pm 0.6^{\mathrm{A}}$ & $30.2 \pm 0.4^{\mathrm{B}}$ & $27.7 \pm 0.9^{\mathrm{A}}$ & $25.7 \pm 1.1^{\mathrm{B}}$ \\
\hline
\end{tabular}

Values of serum lipids are the sum of lipoprotein lipid levels. Liver lipid levels are shown by $\mathbf{M} \pm$ SEM of 7 to 10 quail. Same capital and small superscript letters show statistically significant difference between strains (capital) and diets (small) at $p<$ 0.01 . CA, commercially available Japanese quail; HAP, hyperlipidemic atherosclerosis-prone Japanese quail; - , cholesterol-free;,$+ 1 \%$ cholesterol.

Serum total cholesterol levels were increased 4.5- and 12.6-fold in CA and HAP quail, respectively. Similarly, the increases in serum triglyceride levels were 2.1and 5.1-fold for CA and HAP quail, respectively. The serum cholesterol and triglyceride concentrations of HAP quail fed cholesterol diet were 5.1- and 3.3-fold higher than those of the CA quail, respectively. The dietary cholesterol-dependent increases in the liver total cholesterol levels were 5.3- and 5.5-fold in the CA and HAP quail, respectively. The liver cholesterol levels of the HAP quail were significantly higher than those of the CA quail. No changes of other lipid classes in the liver were observed in either strain. Therefore, it is likely that cholesterol ester might be the major lipid component accumulated in the liver with the feeding of a cholesterol diet.

Figure 1 shows the lipoprotein distribution patterns of both strains fed either the cholesterol-free or cholesterol diets. Lipoprotein patterns were assessed by cholesterol concentration using the density. The lipoprotein patterns of the HAP quail (Fig. 1B) were similar to those of the CA quail (Fig. 1A). In the case of quail 


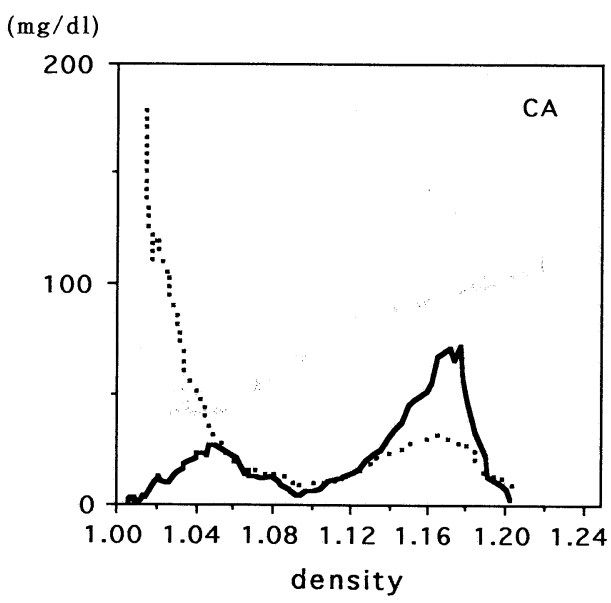

(a) $(\mathrm{mg} / \mathrm{dl})$

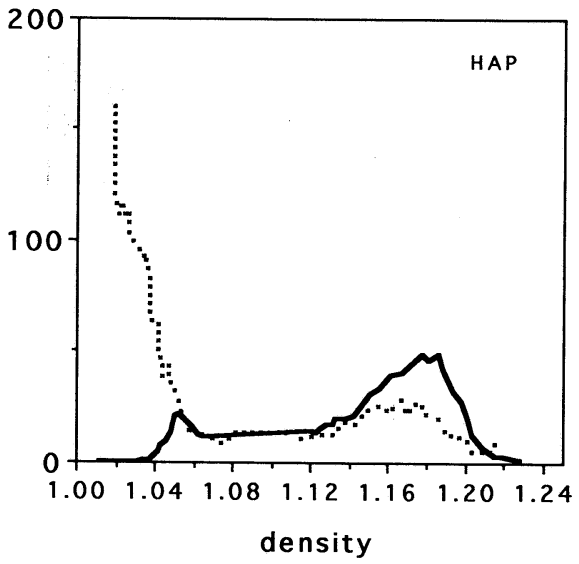

(b)

Fig. 1. Lipoprotein distribution patterns of CA and HAP quail fed $1 \%$ cholesterol or cholesterol-free diets. Cholesterol concentration was plotted against density of successive $0.1 \mathrm{ml}$ fractions. $\cdots, 1 \%$ cholesterol; - , cholesterol-free.

fed the cholesterol-free diet, serum lipoprotein consisted of two peaks: a minor one ranging from $d=1.02$ to $d=1.09 \mathrm{~g} / \mathrm{ml}$ and a major one ranging from $d=1.09$ to $d=$ $1.21 \mathrm{~g} / \mathrm{ml}$. The characteristics of lipoprotein distributions of quail fed the cholesterol diet were manifested in the substantial increase of cholesterol level in the chylomicron and VLDL fractions $(d<1.055)$. Thus, changes induced by cholesterol loading were noted in the low-density fractions $(d<1.055)$ rather than the high-density lipoprotein fractions of HAP quail. In addition, the increase of cholesterol level in the low-density fractions of the HAP quail was much greater than that of the CA quail.

The density cutoff was elicited based on the lipoprotein profiles described in Fig. 1, and was adopted for the fractionation of lipoprotein by cumulative flotation: $d<1.006 \mathrm{~g} / \mathrm{ml}$ for chylomicron, $1.006<d<1.020 \mathrm{~g} / \mathrm{ml}$ for $\mathrm{VLDL}, 1.020<d<1.080$ $\mathrm{g} / \mathrm{ml}$ for $\mathrm{LDL}$, and $1.080<d<1.210 \mathrm{~g} / \mathrm{ml}$ for $\mathrm{HDL}$, respectively. Lipoprotein floated by brief centrifugation at $d<1.006 \mathrm{~g} / \mathrm{ml}$ was tentatively classified as chylomicron in this study. On the feeding of cholesterol, substantial lipoprotein was recovered into this fraction even though animals were starved overnight. Combined samples of serum from the same diet group were used for the isolation of lipoproteins.

The chemical compositions of the lipoprotein fractions in both strains are shown in Fig. 2. No substantial difference between the CA and HAP quail fed cholesterol-free diets was observed. A trace amount of lipids was detectable in the chylomicron of both quail. The feeding of cholesterol increased the level of cholesterol ester, which amounted to more than 50\% in the chylomicron and VLDL fractions of the cholesterol-fed groups. Cholesterol feeding reduced the 
(\%)

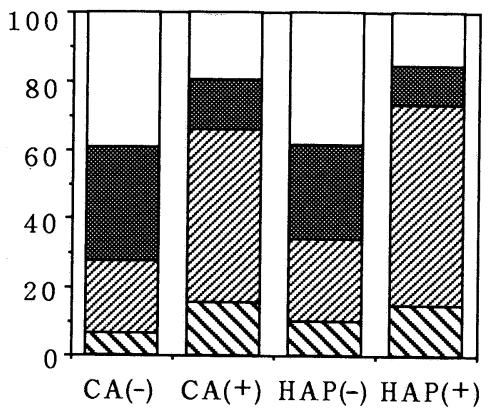

(\%)

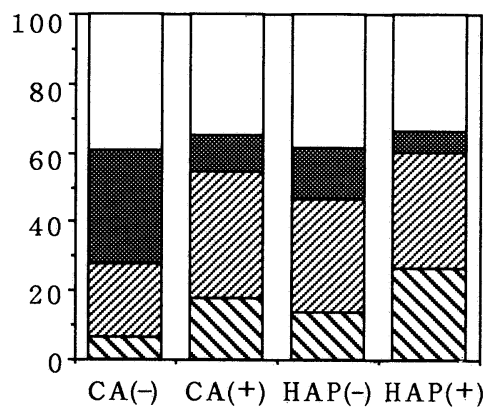

(\%)

VLDL

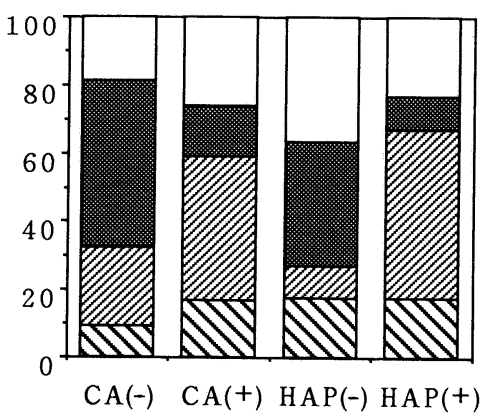

(\%)

HDL

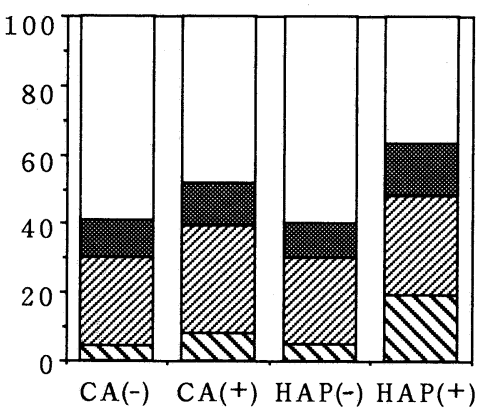

Fig. 2. Chemical composition of lipoprotein fractions isolated from CA and HAP quail fed $1 \%$ cholesterol or cholesterol-free diets. $\mathbb{N}$, free cholesterol; $\mathbb{Z}$, cholesterol ester; 図, triglyceride; $\square$, phospholipid.

level of phospholipid in the HDL fraction slightly $(d>1.08)$. These observations show that chylomicron and VLDL are the major vehicles for the transport of dietary cholesterol in quail. The proportion of cholesterol ester in the lipoproteins of the HAP quail appeared to be greater than that of the CA quail. This appeared to be true especially for chylomicron and VLDL of the cholesterol-fed group.

The separation of both strain lipoproteins by SDS-PAGE is shown in Fig. 3. As described previously (9), the lipoprotein fraction was isolated by cumulative flotation and purified by re-spinning in the same medium. Samples solubilized directly in 20\% SDS solution were applied to 3 and 12\% SDS-PAGE gels. It found that the major component of apoprotein in the HDL fraction was the protein of 26 $\mathrm{kDa}$, whereas in low-density fractions $(d<1.055)$, a protein of molecular weight over $100 \mathrm{kDa}$ comprised the greater proportion. This, however, is not the case for the apoprotein profile of the chylomicron fraction. It is noteworthy that cholesterol feeding elevated the level of higher molecular weight apoprotein $(470 \mathrm{kDa})$ in the lower density of lipoprotein fractions $(d<1.02)$. This picture is accentuated in HAP quail rather than in CA quail (Fig. 4). Only a small amount of apoprotein of 
$(\mathrm{kDa})$

(kDa)

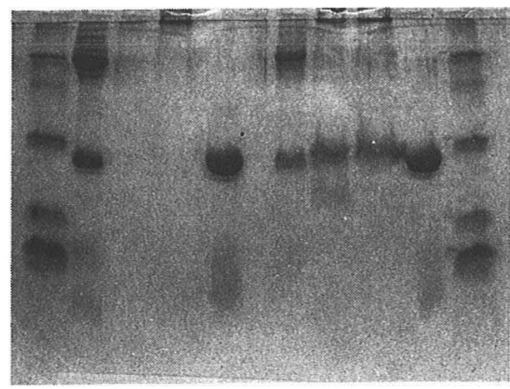

94
67
43
30
20
14

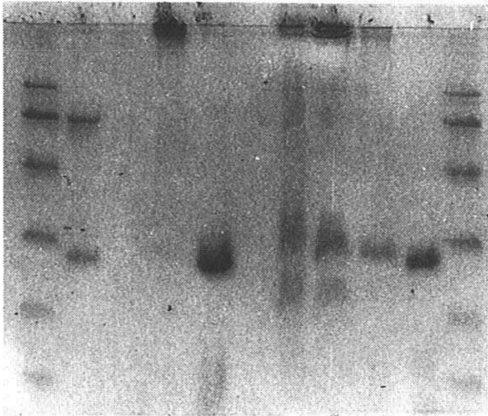

94

67

43

30

20

14

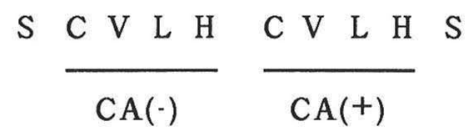

(a)

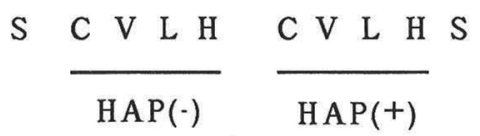

(b)

Fig. 3. 12\% SDS-polyacrylamide gel electrophoresis of isolated lipoprotein fractions from CA (A) and HAP quail (B) fed either 1\% cholesterol or cholesterol-free diets. S, standard protein; C, chylomicron; V, VLDL; L, LDL; H, HDL; denotes cholesterol-free; + denotes $1 \%$ cholesterol.

$(\mathrm{kDa})$

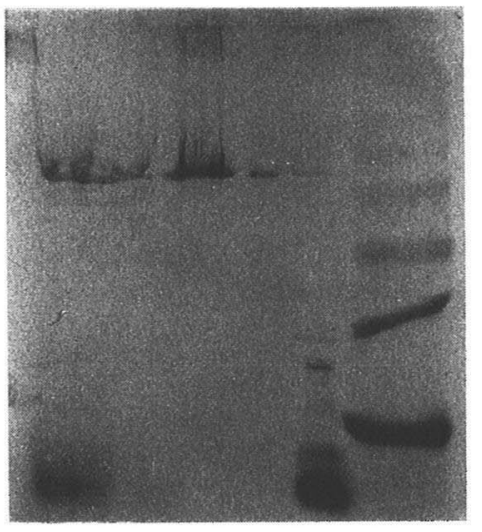

$\mathrm{HAP}(-) \quad \mathrm{HAP}(+)$

Fig. 4. 3\% SDS-polyacrylamide gel electrophoresis of isolated lipoprotein fractions from HAP quail fed either $1 \%$ cholesterol or cholesterol-free diet. S, standard protein; C, chylomicron; V, VLDL; L, LDL; - denotes cholesterol-free; + denotes $1 \%$ cholesterol. 


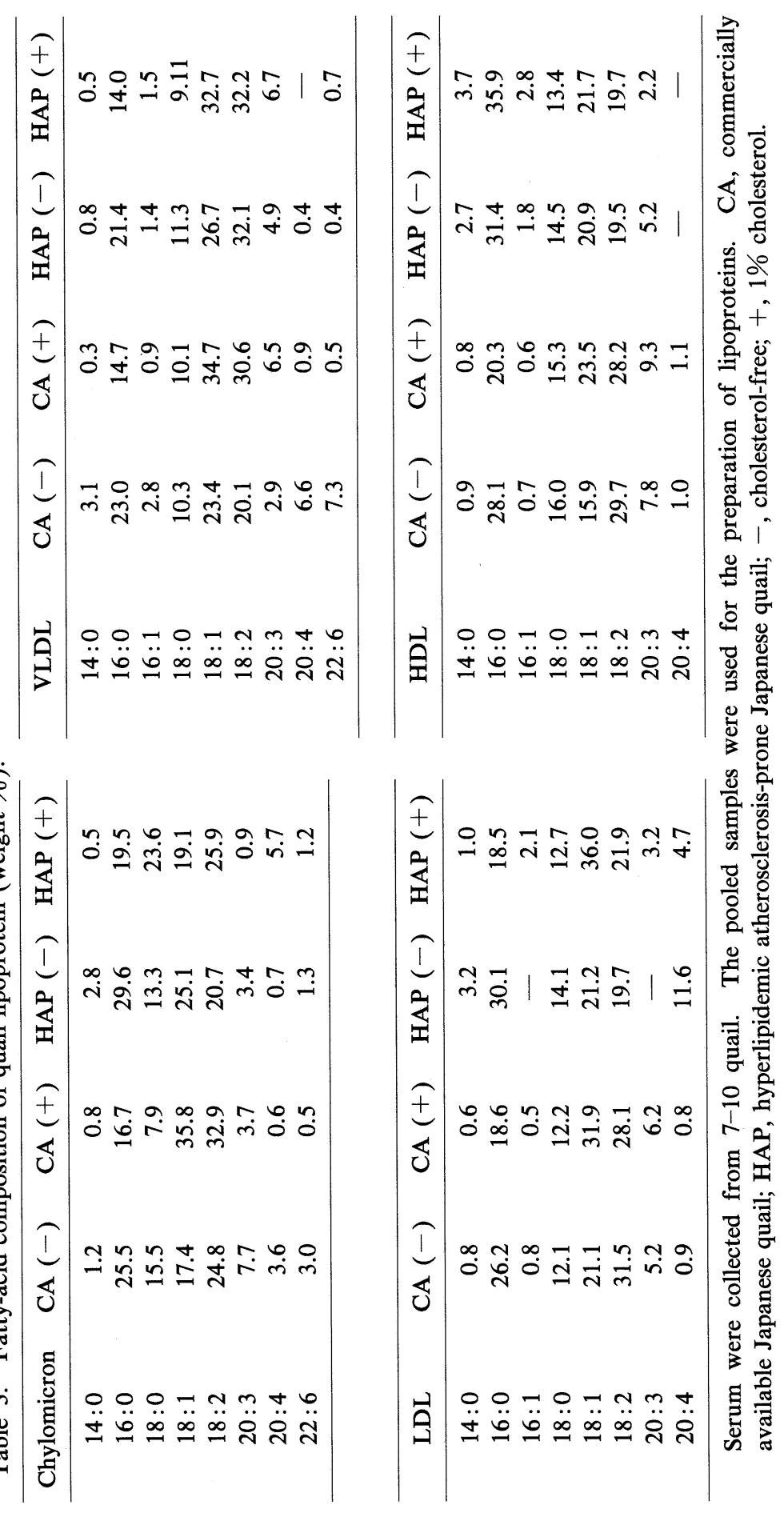


$470 \mathrm{kDa}$ was present in the LDL of CA quail or HAP quail fed the cholesterol-free diet.

Table 3 lists the fatty-acid compositions for pooled samples of lipoproteins. In general, the data for pooled samples well represents the mean value of individual samples. With the feeding of cholesterol, the level of 16:0 decreased in the chylomicron, VLDL and LDL fractions of CA and HAP quail, while the levels of $18: 1$ and $18: 2$, which are known as neutral or anti-atherogenic fatty acids, tended to increase in VLDL. Therefore, it seems to be true that cholesterol feeding causes an alteration in the fatty-acid profiles of chylomicron, VLDL and LDL but not in the HDL fraction.

\section{DISCUSSION}

In this investigation, comparisons of the lipoprotein profiles were made between HAP and CA quail fed diets with and without cholesterol. Our results show a marked increase in cholesterol ester content of low-density lipoprotein fractions $(1.006<d<1.055)$ and a slight alteration in the chemical composition of highdensity lipoprotein fractions $(1.08<d)$. This study also noted the presence of high molecular weight apoprotein $(470 \mathrm{kDa})$ in the low-density fraction $(d<1.055)$ of HAP quail.

It is the general view that cholesterol feeding alters the lipid profiles of the VLDL and LDL fractions rather than those of the HDL fraction, and leads to atheroma formation in many species $(12-14)$. HDL acts as an anti-atherosclerotic lipoprotein and an effective substrate for LCAT reaction $(15,16)$. We indicated in a previous report (9) that the major protein component of quail lipoprotein is apo A-I. It is also well-known that this apoprotein activates LCAT reactivity and promotes the esterification of cholesterol in humans (17). The alteration of lipoprotein and apoprotein profiles induced by cholesterol intake may affect the interaction between lipoprotein fractions, which may consequently modulate the accumulation of cholesterol in the serum. Therefore, elucidation of the correlation between the catabolism by enzymes such as lipoprotein lipase (LPL), cholesterol ester transfer protein (CETP) or LCAT and the lipid profiles of various lipoprotein fractions might shed light on the hypercholesterolemia of HAP quail.

In this study, of greater interest was the observation of a high molecular weight apoprotein $(470 \mathrm{kDa})$ in the low-density lipoprotein fractions $(d<1.055)$ of HAP quail. This protein apparently corresponds to chicken apo B-100 $(450 \mathrm{kDa})$, which is a major apolipoprotein in the lipoproteins of $d<1.046 \mathrm{~g} / \mathrm{ml} \mathrm{(18).} \mathrm{Thus,} \mathrm{the}$ increase of cholesterol concentration in low-density fractions resulted in the existence of a high molecular weight apoprotein in HAP quail. On the other hand, the moderate cholesterol increase in CA quail was not accompanied by the increased expression of a carrier protein for cholesterol disposal. Therefore, it appears plausible that the presence of a high molecular apoprotein in lower density fractions may be needed for cholesterol transport and metabolism via LDL receptor in quail 
fed a cholesterol diet. In addition, we observed that the apo A-I mRNA level of the quail liver and intestine was elevated by cholesterol feeding (manuscript in preparation). Taking these facts into consideration, it may be possible that the presence of excess cholesterol would elevate apo B-100 synthesis for the assembly of VLDL.

The incorporation of serum cholesterol by LDL receptor-mediated endocytosis is of major importance for cholesterol homeostasis. The receptor is present in the liver parenchymal cells, and recognizes the apoprotein B-100 component of LDL (19). The major transport protein for endogenous cholesterol in plasma is LDL. The interaction of LDL receptor and apoprotein B-100, therefore, may play an important role in receptor-mediated cholesterol homeostasis.

Recently, Elkin et al. suggested the presence of a cell-specific receptor for VLDL on the oocyte of quail, and characterized this receptor (20). The receptor plays a biological role for vitellogenesis, which brings about massive uptake of yolk precursors from the serum. From this point of view, it is possible that the uptake of cholesterol may be regulated by the function of endocytosis of quail liver parenchymal cells. Therefore, further study is needed to elucidate whether the receptor-mediated uptake of cholesterol by quail liver parenchymal cells bears some relevance to the genesis of hypercholesterolemia and cholesterol homeostasis in HAP quail.

The authors thank Otsuka Pharmaceutical Co., Ltd. for supplying HAP Japanese quail. Our thanks also go to K. Iwatani for technical assistant.

\section{REFERENCES}

1) Keys, A. (1975): Coronary heart disease - the global picture. Atherosclerosis, 22, 149192.

2) Dangerfield, W. G., Finlayson, R., Myatt, G., and Mead, M. G. (1976): Serum lipoproteins and atherosclerosis in animals. Atherosclerosis, 25, 95-106.

3) Kushwaha, R. S., Reardon, C. A., Getz, G. S., Lewis, D. S., Rice, K. S., Carey, K. D., and McGill, H. C., Jr. (1994): Metabolic mechanisms for responses to dietary cholesterol and fat in high and low LDL responding baboons (Papio sp.). J. Lipid Res., 35, 633-643.

4) Philips, M. C., Johnson, W. J., and Rothblat, G. H. (1987): Mechanism and consequences of cellular cholesterol exchange and transfer. Biochim. Biophys. Acta, 906, 223-276.

5) Illingworth, D. R., Portman, O.W., Robertson, A. L., and Magyar, W. A. (1973): The exchange of phospholipids between plasma lipoproteins and rapidly dividing human cells grown in tissue culture. Biochim. Biophys. Acta, 306, 422-436.

6) Brown, M. S., and Goldstein, J. L. (1986): A receptor-mediated pathway for cholesterol homeostasis. Science, 232, 34-47.

7) Toda, T., Fukuda, N., Oku, H., Terada, K., Nagata, J., Wada, K., and Chinen, I. (1994): Evaluation of atherogenecity of dietry fat in Japanese quail by principal component analysis. J. Jpn. Soc. Nutr. Food Sci., 47, 401-405. 
8) Wu, T.-C., and Donaldson, W. E. (1982): Effect of cholesterol feeding on serum lipoproteins and atherosclerosis in atherosclerosis-susceptible and atherosclerosisresistant Japanese quail. Poultry Sci., 61, 2407-2414.

9) Oku, H., Ishikawa, M., Nagata, J., Toda, T., and Chinen, I. (1993): Lipoprotein and apoprotein profile of Japanese quail. Biochim. Biophys. Acta, 1167, 22-28.

10) Oku, H., Fukuda, N., Mori, M., Kinjyo, M., Motono, H., and Chinen, I. (1988): Hypocholesterolemic effect of bagasse alkaline extract in rats fed atherogenic diet. Nutr. Rep. Int., 38, 843-852.

11) George, W. S., and William, G. C. (1980): Two-way tables with unequal numbers and proportions, in Statistical Methods, 7th ed., The Iowa State University Press, Iowa, pp. 414-433.

12) Kwiterovich, P. O., Jr., and Sniderman, A. D. (1983): Atherosclerosis and apoproteins B and A-I. Prev. Med., 12, 815-835.

13) Kukita, H., Hiwada, K., and Kokubo, T. (1984): Serum apolipoprotein A-I, A-II and B levels and their discriminative values in relatives of patients with coronary artery disease. Atherosclerosis, 51, 261-267.

14) McGill, H. C., Jr., McMahan, C. A., Kuruski, A. W., and Mott, G. E. (1981): Relationship of lipoprotein cholesterol concentrations to experimental atherosclerosis in baboon. Atherosclerosis, 1, 3-12.

15) Castelli, W. P., Doyle, J. T., Gordon, T., Hames, C. G., Hjortland, M. C., Hulley, S. B., Kagan, A., and Zukel, W. J. (1977): HDL cholesterol and other lipids in coronary heart disease. The cooperative lipoprotein phenotyping study. Circulation, 55, 767772.

16) Pieters, M. N., Schouten, D., and Van Berkel, Th. J. C. (1994): In vitro and in vivo evidence for the role of HDL in reverse cholesterol transport. Biochim. Biophys. Acta, 1225, 125-134.

17) Fielding, C. J., Shore, V. G., and Fielding, P. E. (1972): A protein cofactor of lecithin: cholesterol acyltransferase. Biochem. Biophys. Res. Commun., 46, 1493-1498.

18) Herier, D., Forgez, P., and Chapman, M. J. (1985): A density gradient study of the lipoprotein and apolipoprotein distribution in the chicken, Gallus domesticus. Biochim. Biophys. Acta, 836, 105-118.

19) Chao, Y.-S., Windler, E. E., Chen, G. C., and Havel, R. J. (1979): Hepatic catabolism of rat and human lipoproteins in rats treated with $17 \alpha$-ethinyl estradiol. J. Biol. Chem., 254, 11360-11366.

20) Elkin, R. G., MacLachlan, I., Hermann, M., and Schneider, W. J. (1995): Characterization of the Japanese quail oocyte receptor for very low density lipoprotein and vitellogenin. J. Nutr., 125, 1258-1266. 